\title{
A New Computer Diagnostic System for Ventricular and Supraventricular Arrhythmias
}

\author{
by Dongring Lin, Ph.D., ${ }^{*}$ Janice M. Jenkins, Ph.D., ${ }^{*}$ and Lorenzo A. DiCarlo, M.D.†
}

A new arrhythmia detection system has been designed and successfully tested on two separate databases that consist of simultaneous atrial and ventricular electrograms. The system examines the $P$ wave and QRS morphologies, timing information, and the conduction sequences and derives a diagnosis of arrhythmias on a beat-by-beat basis. It also diagnoses the underlying rhythm by examining the eight most current beats. One database has an esophageal electrogram for atrial information and a surface electrocardiogram (lead II) for ventricular information recorded from patients in coronary intensive care. The other one has intraatrial and intraventricular electrograms recorded from catheter electrodes during electrophysiology studies.

Atrial and ventricular morphologies are analyzed by correlation waveform analysis. An underlying conduction sequence is derived from the morphologic measurement and timing information. For any combination of incoming atrial depolarization (A) and ventricular depolarization (V), a conduction sequence is assigned to one of four classes: blocked atrial depolarization, atrialventricular depolarization, blocked ventricular depolarization, or ventricular-atrial depolarization. A beat code consisting of AA, AV, and VV intervals and $A$ and $V$ morphologic indices is then generated for the incoming beat.

Test results on the esophageal-surface data

\footnotetext{
* From the School of Engineering and + St. Joseph Mercy Hospital and the School of Medicine, University of Michigan, Ann Arbor, Michigan.

Supported by a grant from the Whitaker Foundation, NSF Grants ECS-8351215 and ECS-8506494, NIH Grant RO1 HL35554, a grant from the office of Vice President for Research, and Rackham Graduate School, University of Michigan.

Reprint requests to: Lorenzo DiCarlo, M.D., Cardiology Associates of Ann Arbor, Reichert Health Building, Suite 3003, 5333 McAuley Drive, Ypsilanti, MI 48197.
}

base yielded $99.2 \%$ accuracy for single-beat diagnosis and $99.5 \%$ accuracy for contextual diagnosis. The system also successfully diagnoses some arrhythmias that cannot be reliably diagnosed by any other system, such as sinus beats with aberrant conduction, sinus beats with AV delay, premature atrial contractions with or without conduction, premature ventricular contractions with or without retrograde activation, atrial ectopic beats with normal or abnormal conduction, atrial ectopic beats with or without conduction, atrial bigeminy, first-degree AV block, second-degree AV block, ventricular couplets with $1: 1$ retrograde, supraventricular tachycardia with $1: 1$ and $2: 1$ conduction, atrial flutter with $2: 1$ conduction, ventricular tachycardia without retrograde, and ventricular tachycardia with $1: 1$ and 2:1 retrograde. Successful results on the esophageal-surface date base suggest that the system can provide reliable arrhythmia monitoring in a noninvasive manner.

Test results on the intracardiac data base demonstrated $97.1 \%$ accuracy for both single-beat and contextual diagnoses. Some arrhythmias that cannot be reliably diagnosed by any other systems were successfully diagnosed, such as atrial couplets with 2:1 conduction. Results on the intracardiac database show promise in improved rhythm analysis for applications such as implantable antitachycardia devices. 